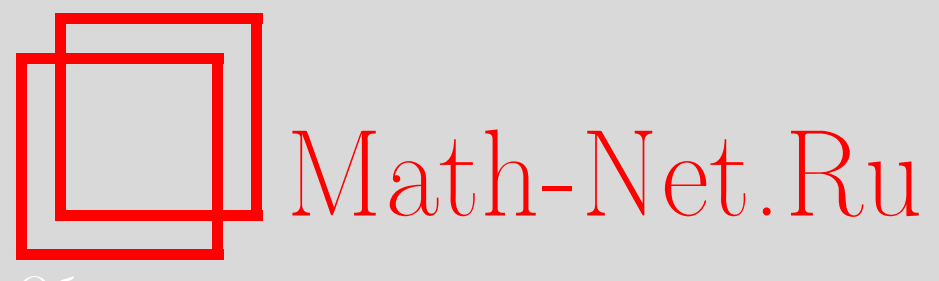

Б. А. Рогозин, Компактность и функции концентрации сверток распределения, Теория вероятн. и ее примен., 2005, том 50, выпуск 1, 81-97

DOI: https://doi.org/10.4213/tvp159

Использование Общероссийского математического портала MathNet.Ru подразумевает, что вы прочитали и согласны с пользовательским соглашением

http://www . mathnet.ru/rus/agreement

Параметры загрузки:

IP : 34.239 .49 .27

26 апреля 2023 г., $16: 37: 38$

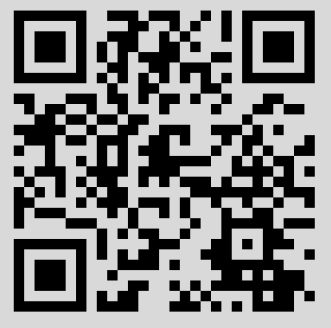




\section{КОМПАКТНОСТЬ И ФУНКЦИИ КОНЦЕНТРАЦИИ СВЕРТОК РАСПРЕДЕЛЕНИЯ ${ }^{1)}$}

Исследуется связь между условиями компактности распределений сумм независимых одинаково распределенных случайных величин при подходящей нормировке и поведением последовательности функций концентрации этих сумм.

Ключевые слова и фразы: независимые одинаково распределенные случайные величины, сумма, свертка, медиана, квантиль, компактность, конечная некомпактность, функция концентрации Леви, сенсорная дисперсия, мажорируемо меняющаяся функция, одновершинное распределение.

1. Определения, формулировки теорем. Рассмотрим последовательность независимых одинаково распределенных случайных величин $X, X(1), X(2), \ldots$. Положим $S(n)=X(1)+\cdots+X(n), F^{(n)}-$ распределение $S(n), \mu(n)=\mu(S(n))$ - медиана $S(n)$. В работах [1] и [2] содержатся условия, обеспечивающие существование последовательности положительных чисел $\{b(n), n=1,2, \ldots\}$, для которой совокупность распределений случайных величин $(S(n)-\mu(n)) / b(n)$ компактна, т.е. для любого $\varepsilon>0$ существует $x_{0}$ такое, что $\mathbf{P}\left\{|S(n)-\mu(n)|>x_{0} b(n)\right\}<\varepsilon$ при всех $n$. В частности, для этого необходимо и достаточно, чтобы

$$
\limsup _{x \rightarrow \infty} \frac{x^{2} \mathbf{P}\{|X|>x\}}{\mathbf{E}\left(X^{2} ;|X| \leqslant x\right)}<\infty
$$

Эти результаты были дополнены в работе [3] исследованием поведения сенсорной дисперсии распределения $X$ (см. [4, п. 2.2.2])

$$
w(x)=x^{-2} \int_{0}^{x} y \mathbf{P}\{|X|>y\} d y=\frac{1}{2} \mathbf{E} \min \left\{\left(\frac{X}{x}\right)^{2}, 1\right\}, \quad x>0,
$$

и поведения последовательности функций концентрации $Q(n)=$ $Q\left(h, F^{(n)}\right)=\sup _{y} \mathbf{P}\{y \leqslant S(n) \leqslant y+h\}$ при фиксированном $h>0$. Приведем эти результаты с некоторыми дополнениями. Введем необходимые

* Омский филиал Института математики им. С. Л. Соболева СО РАН, ул. Певцова, 13, 644099 Омск, Россия.

1) Работа выполнена при поддержке Российского фонда фундаментальных исследований (проекты 99-01-01130, 00-15-96178) и INTAS (проект 00-265). 
обозначения и определения. Положим $d(u)=w(1 / u)$ при $u>0, d(0)=0$, обратная $d$ функция $D(t)=l(u \geqslant 0: d(u) \leqslant t)$ при $t \geqslant 0$, где $l-$ мера Лебега на прямой.

Функция $B$ называется надстепенной в 0 возрастающей функцией (н. в 0 в. ф.), если $B-$ положительная и монотонно неубывающая при $t>0$ функция и $\lim \sup _{t \rightarrow 0} B(t) / B(\lambda t)<\infty$ при некотором $\lambda(0<\lambda<1)$; функцию $A(x)=B(1 / x)$ будем называть надстепенной на бесконечности убывающей функцией (н. на б. у. ф.). Последний класс функций совпадает с совокупностью мажорируемо меняющихся функций, свойства которых обсуждаются в [5, приложение]. Близкий класс надстепенных функций вводится в монографии [6, гл. 4, §21, п. 4], это название и используется нами в настоящей работе.

Для последовательностей $\{a(n), n=1,2, \ldots\}$ и $\{b(n), n=$ $1,2, \ldots\}$ эквивалентность $a(n) \simeq b(n), n \rightarrow \infty$, означает, что $\limsup _{n \rightarrow \infty} a(n) / b(n)<\infty, \lim \sup _{n \rightarrow \infty} b(n) / a(n)<\infty$. Это обозначение эквивалентности с сохранением смысла в дальнейшем будет также использоваться и для функций.

Теорема 1. Утверждения 1)-3) выполняются одновременно:

1) справедливо неравенство (1);

2) $D(t)-$ н. в 0 в. $\phi$;

3) $Q(n) \simeq D(1 / n), n \rightarrow \infty$, при ююбом фиксированном $h>0$.

Одновременное выполнение неравенства (1) и утверждения 2) теоремы 1 , а также то, что при этом выполняется утверждение 3 ), установлено в [3].

Обозначим $v(t)=1-|f(t)|, f(t)=\mathbf{E} \exp \{i t X\}$. Функция $g$ называется подстепенной в 0 возрастающей функцией (п. в 0 в. ф.), если $g$ положительна и монотонно не убывает при $t>0$ и $\lim \sup _{t \rightarrow 0} g(\lambda t) / g(t)<1$ при некотором $\lambda(0<\lambda<1)$ (см. [7]).

Теорема 2. Вместе с неравенством (1) одновременно выполняются следуюшие соотношения и утверждения:

1) $d(t)-n$. в 0 в. $\phi$;

2) $v(t) \simeq a(t), t \rightarrow 0$, где $a-n$. в 0 в. $\phi$;

3) $v(t) \simeq b(t), t \rightarrow 0$, где $b-$ монотонно неубывающая положительная функция при $t>0, u \lim \sup _{n \rightarrow \infty} Q(n) / Q(m n)<\infty$ при некотором $m \in\{2,3, \ldots\}$.

Связь между надстепенным убыванием последовательности $\{Q(n)$, $n=1,2, \ldots\}$ и поведением характеристической функции в 0 устанавливается в следующем предложении. Обозначим $V(u)=l\{t: v(t) \leqslant u, 0<$ $t<z\}$ при $u>0$ и некотором фиксированном $z>0$ таком, что $v(t)>0$ при $0<t \leqslant z$.

Теорема 3. Следуюшие соотношения и утверждения выполняются одновременно: 
1) $V(u)-$ H. в 0 в. $\oint$;

2) $\limsup _{n \rightarrow \infty} Q(n) / Q(m n)<\infty$ при любом (некотором) $m=$ $2,3, \ldots$;

3) $Q(n) \simeq V(1 / n), n \rightarrow \infty$, при любом биксированном $h>0$.

В соответствии с п. 3) теоремы 2 выполнение неравенства (1) эквивалентно условиям, что функция $v(t)$ почти монотонна в 0 (или, что равносильно, $v(t) \simeq d(t), t \rightarrow 0)$ и последовательность $\{Q(n), n=1,2 \ldots\}$ является надстепенной убывающей последовательностью. Почти монотонное в 0 возрастание функции $v$ не приводит к выполнению неравенства (1), и потому возникает вопрос, не будет ли из надстепенного убывания последовательности $\{Q(n), n=1,2 \ldots\}$ следовать выполнение неравенства (1). Тем более что в [8, теорема 3] доказывается, что если

$$
\lim _{m \rightarrow \infty} \frac{1}{m} \limsup _{n \rightarrow \infty} \frac{Q(n)}{Q(m n)}=0
$$

то выполняется (1).

Для того чтобы сформулировать теорему, связанную с этим вопросом, введем необходимые обозначения и определения. Пусть $K\left(p, F^{(n)}\right)=$ $K(p, n), \quad 0<p<1, n=1,2, \ldots$, есть $p$-квантиль распределения $|S(n)-\mu(n)|: \mathbf{P}\{|S(n)-\mu(n)|<K(p, n)\} \leqslant p \leqslant \mathbf{P}\{|S(n)-\mu(n)| \leqslant K(p, n)\}$.

Будем говорить, что совокупность сверток $\left\{F^{(n)}, n=1,2, \ldots\right\}$ имеет порядок некомпактности, не превосходящий $k$ (не больший $k$ ), если для любой последовательности целых чисел $\{m(j), j=1,2, \ldots\}$ и любого набора $0<p_{1}<\cdots<p_{l}<1, l \geqslant k+1$, последовательность распределений случайных величин $(S(m(j))-\mu(m(j))) / K\left(p_{i}, m(j)\right)$ при $j \rightarrow \infty$ слабо сходится к предельному, возможно несобственному, распределению $G_{i}$, $i=1, \ldots, l$, и среди чисел $G_{1}(\mathbf{R}), \ldots, G_{l}(\mathbf{R})$ не более $k$ различных, меньших 1 .

Отметим, что $0<G_{1}(\mathbf{R}) \leqslant \cdots \leqslant G_{l}(\mathbf{R}) \leqslant 1$, и потому существует набор $p_{i_{1}}, \ldots, p_{i_{r}}$ такой, что $0<G_{i_{1}}(\mathbf{R})<\cdots<G_{i_{r}}(\mathbf{R}) \leqslant 1$, а остальные значения $G_{i}(\mathbf{R})$ или совпадают с одним из чисел $G_{i_{1}}(\mathbf{R}), \ldots, G_{i_{r}}(\mathbf{R})$, или равны $1, r \leqslant k$. В частности, если порядок некомпактности не превосходит 0 , то из любой последовательности целых чисел $\{n(j), j=1,2, \ldots\}$ можно извлечь подпоследовательность $\{m(j), j=1,2, \ldots\}$, для которой последовательность распределений случайных величин $(S(m(j))-$ $\mu(m(j))) / K\left(\frac{1}{2}, m(j)\right)$ слабо сходится при $j \rightarrow \infty$ к невырожденному вероятностному распределению, и потому выполняется неравенство (1).

С помощью предельной теоремы для отношений функций концентрации [9] можно показать (см. лемму 5 ниже), что если выполнено соотношение 2) теоремы 3 , то любая монотонная функция $q(x)$, для которой $q(n)=Q(n)$ при $n=1,2, \ldots$, будет н. на б. у. ф. Применяя рассуждения доказательства леммы П4 из [5] к функции $B(y)=$ 
$\limsup _{x \rightarrow \infty} A(x y) / A(x)$ при $y>1$, где $A(x)=1 / q(x)$, получим, что $\ln B\left(e^{t}\right)=: H(t)$ при $t>0$ есть полуаддитивная конечная функция, и потому существует конечный предел $\alpha=\lim _{t \rightarrow \infty} H(t) / t \geqslant 0$; ниже будет показано (см. лемму 11), что $\alpha \geqslant \frac{1}{2}$. Число $\alpha$ называется (см. [10]) (асимптотическим) порядком изменения $A(x)$, и потому - $\alpha$ будем называть (асимптотическим) порядком надстепенного изменения функции $q(x)$, а также последовательности $\{Q(n), n=1,2, \ldots\}$.

Теорема 4. Если порядок надстепенного изменения последовательности $\{Q(n), n=1,2, \ldots\}$ равен $-\alpha$, то последовательность сверток $\left\{F^{(n)}, n=1,2, \ldots\right\}$ имеет порядок некомпактности, не больuuน $[2 \alpha-1]$.

Из соотношения (2) и леммы 12 вытекает, что в этом случае $\alpha<1$, и потому порядок некомпактности $\left\{F^{(n)}, n=1,2, \ldots\right\}$ равен 0 , т.е. из теоремы 4 вытекает теорема 3 работы [8]. Приведем еще класс распределений, для которых выполнение неравенства (1) эквивалентно условию, что $\{Q(n), n=1,2, \ldots\}$ - надстепенная убывающая последовательность.

Следствие. Если $F^{(1)}$ - одновершинное распределение, то неравенство (1) выполняется одновременно с утверждением $n$. 2) теоремьз 3 .

2. Доказательство теорем 1-3. Приведем необходимые для дальнейшего сведения из [7]. Пусть $F(t)$ - монотонно неубывающая и ограниченная на $[0, \infty)$ функция, $\lim _{t \rightarrow 0} F(t)=F(0)=0, F(t)>0$ при $t>0$ и $\widehat{F}(x)=\int_{0}^{\infty} \exp \{-x t\} d F_{1}(t)$, где $F_{1}(t)=\sup _{0 \leqslant u<t} F(u)$ при $t>0$, $F_{1}(0)=0$. Следующая теорема совпадает с теоремой 1 работы [7].

Теорема 5. Утверждения 1)-3) выполняются одновременно:

1) $F(t)-$ H. в 0 в. $\oint$;

2) $\widehat{F}(t)-$ н. на б. $у . \oint . ;$

3) $F(t) \simeq \widehat{F}(c / t), t \rightarrow 0$, при некотором (всех) $c>0$.

Введем для неотрицательных функций $a(t)$ и $b(t)$ отношение мажорирования: $a(t) \preceq b(t), t \rightarrow 0$, если $\limsup _{t \rightarrow 0} a(t) / b(t)<\infty$. Аналогичный смысл имеет это обозначение отношения мажорирования для последовательностей, а также функций при $x \rightarrow \infty$. В дальнейшем условимся, что в отношениях эквивалентности и мажорирования переменные $x, y$ и $n$ стремятся к бесконечности, а $u$ и $t-\mathrm{k} 0$, и потому будем опускать указания, при каком предельном переходе имеют место эти отношения.

Лемма 1. При любой постоянной $c>0 F(t) \preceq \widehat{F}(c / t)$.

Это утверждение совпадает с леммой 3 из [7].

Лемма 2. Если $F(t) \simeq G(t) u G(t)-$ n. в 0 в. $\oint$., mо $F(t)-$ n. в 0 в. $\oint$. 
Для надстепенной функции $G$ аналогичное утверждение следует непосредственно из определения.

Д о к а з а т е л ь с т в о. Воспользуемся неравенством леммы 2 из работы [7]. Для п. в 0 в. ф. $G$ существуют постоянные $C>0, t_{0}>0$, $\alpha>0$ такие, что $G(\lambda t) / G(t) \leqslant C \lambda^{\alpha}$ при $0<t<t_{0}$ и $0<\lambda<1$, и так как $C_{1} G(t) \leqslant F(t) \leqslant C_{2} G(t)$ при $0 \leqslant t \leqslant t_{1}$ для некоторых постоянных $t_{1}>0, C_{1}>0, C_{2}>0$, то $F(\lambda t) / F(t) \leqslant C_{2} G(\lambda t) /\left(C_{1} G(t)\right) \leqslant C C_{2} \lambda^{\alpha} / C_{1}$ при $0<t \leqslant t_{2}=\min \left(t_{0}, t_{1}\right)$.

Поэтому при достаточно малом $\lambda(0<\lambda<1)$ отношение $F(\lambda t) / F(t)$ для $0<t \leqslant t_{2}$ будет меньше 1 , и, следовательно, $F-$ п. в 0 в. ф.

Пусть $a(t)$ - непрерывная или монотонно неубывающая неотрицательная функция при $0 \leqslant t \leqslant z, \lim _{t \rightarrow 0} a(t)=0=a(0), a(t)>0$ при $0<t \leqslant z$ и $A(u)=l(t: a(t)<u, 0 \leqslant t \leqslant z)$ при $u>0, A(0)=0$. Пусть $b(t)$ обладает этими же свойствами и $B(u)$ определяется аналогичным образом.

Лемма 3. Если $a(t) \preceq b(t)$, mo $B(t) \preceq A(C t)$ nри некоторой постоянной $C>0$. В частности, если $a(t) \simeq b(t)$, то $B\left(C_{1} t\right) \preceq A(t) \preceq B\left(C_{2} t\right)$ при некоторых постоянных $C_{1}>0 u C_{2}>0$.

Д ок аз а т ельст в о. Если $a(t) \preceq b(t)$, то при $0<t<t_{1}$ справедливо неравенство $C_{1} a(t) \leqslant b(t)$, где $C_{1}>0-$ некоторая постоянная. Отсюда вытекает, что $\left\{t: C_{1} a(t)<u, 0<t \leqslant t_{1}\right\} \supset$ $\left\{t: b(t)<u, 0<t \leqslant t_{1}\right\}$. Выберем значение $t_{0} \leqslant t_{1}$ достаточно малым, чтобы выполнялось неравенство $u_{0}=\max _{0<t \leqslant t_{0}} b(t)<$ $\min \left\{\min _{t_{1} \leqslant t \leqslant z} a(t), \min _{t_{1} \leqslant t \leqslant z} b(t)\right\}$. Это возможно благодаря свойствам непрерывности или монотонности функций $a$ и $b$. Поэтому при $0<u \leqslant u_{0}$ имеет место включение $\left\{t: C_{1} a(t)<u, 0<t \leqslant z\right\} \subset$ $\{t: b(t)<u, 0<t \leqslant z\}$, откуда получаем при $0<u<u_{0}$ неравенство $B(u) \leqslant A\left(u / C_{1}\right)$, т.е. утверждение леммы.

Приведем необходимые утверждения, связанные с функциями концентрации. Пусть $q(x), x \geqslant 0,-$ монотонная функция, для которой $q(n)=Q(n), n=1,2, \ldots$.

Лемма 4. Пусть $Q(n) \preceq b(n)$ (соответственно $b(n) \preceq Q(n)$ или $b(n) \simeq Q(n))$ и $b(x), x \geqslant 0,-$ монотонная положительная функиия. Тогда $q(x) \preceq b(x)$ (соответственно $b(x) \preceq q(x)$ или $q(x) \simeq b(x))$.

Д ок аз а тель с т в о. Пусть $q_{1}(x)=Q([x])$ при $x \geqslant 1, q_{2}(x)=$ $Q([x]+1)$ при $[x] \neq x \geqslant 1$ и $q_{2}(n)=Q(n), n=1,2, \ldots$ Из предельной теоремы для отношений функций концентрации (см. [9]) вытекает, что $\lim _{x \rightarrow \infty} q_{1}(x) / q_{2}(x)=1$. Следовательно, все функции $q$ входят в один класс эквивалентных функций. Для $x$ таких, что $n \leqslant x<n+1$, $n=1,2, \ldots$, положим $q_{3}(x)=\max \{Q(n) b(x) / b(n), Q(n+1)\}$; если $Q(n) \preceq b(n)$, то $q_{3}(x) \preceq b(x)$, и, следовательно, $q(x) \preceq b(x)$ для любой функции $q$. Если определить $q_{4}(x)=\min \{Q(n), Q(n+1) b(x) / b(n+1)\}$ 
для $x$ таких, что $n<x \leqslant n+1, n=1,2, \ldots$, и $q_{4}(1)=Q(1)$ и если $b(n) \simeq Q(n)$, то $b(x) \simeq q_{4}(x)$, а следовательно, $b(x) \simeq q(x)$. Случай $Q(n) \simeq b(n)$ сводится к рассмотренным.

Лемма 5. Если $Q(n) \preceq Q(n m)$ при некотором $m \in\{2,3, \ldots\}$, то $q-$ н.наб. $у . \oint$.

Д о к а з а т е л ь с т в о. Если $Q(n) \preceq Q(n m)$ при некотором целом $m \geqslant 2$, то, применяя лемму 4 с $b(x)=q(x m)$, получим, что $q(x) \preceq q(x m)$ с этим же $m$, а следовательно, $q-$ н. на б. у. ф.

Обозначим $g(t)=-\ln |f(t)|$ (заметим, что $g(t)>0$ при $0<t \leqslant z$ ). Пусть также $G(u)=l\{t: g(t)<u, 0 \leqslant t \leqslant z\}, u \geqslant 0$, и $\widehat{G}(x)=$ $\int_{0}^{\infty} \exp \{-x u\} d G(u), x \geqslant 0$.

Лемма 6. Справедливь следуюшие отношения мажорирования: $\widehat{G}(2 n) \preceq Q(n) \preceq \widehat{G}(n), \widehat{G}(2 x) \preceq q(x) \preceq \widehat{G}(x)$.

Д о к а з а т е ль с т в о. Так как $\widehat{G}(x)=\int_{0}^{z}|f(t)|^{x} d t$, то из известных оценок функций концентрации (см. [11, гл. III, §1]) вытекает $\widehat{G}(2 n) \preceq Q\left(1 / z, F^{(n)}\right) \preceq \widehat{G}(n)$, откуда с помощью предельной теоремы для отношений функций концентрации [9] получаем оценки при любом $h>0$, а с помощью леммы 4 - для $q$.

Лемма 7. При любом фиксированном $a w(x) \simeq \int_{0}^{x} y \mathbf{P}\{|X-a|>$ $y\} d y / x^{2}$.

Д ок аз а т ель с т в о. При $x \geqslant x_{0}\left(x_{0} \geqslant 2|a|, w\left(x_{0}\right)>0\right)$

$$
\begin{array}{rl}
\int_{0}^{x} & y \mathbf{P}\{|X-a|>y\} d y \\
& =\int_{0}^{2|a|} y \mathbf{P}\{|X-a|>y\} d y+\int_{2|a|}^{x} y \mathbf{P}\{|X-a|>y\} d y \\
& \leqslant C_{1}+\int_{0}^{x} y \mathbf{P}\left\{|X|>\frac{y}{2}\right\} d y \leqslant C_{2} \int_{0}^{x} y \mathbf{P}\{|X|>y\} d y
\end{array}
$$

где $C_{1}>0$ и $C_{2}>0$ - некоторые постоянные. 'Меняя местами случайные величины $|X-a|$ и $|X|$, получим неравенство $\int_{0}^{x} y \mathbf{P}\{|X|>y\} d y \leqslant$ $C_{3} \int_{0}^{x} y \mathbf{P}\{|X-a|>y\} d y$ при $x \geqslant x_{1}$, откуда и следует утверждение леммы.

Обозначим через $X_{s}$ симметризацию случайной величины $X$ и положим

$$
\begin{gathered}
v_{s}(t)=1-|f(t)|^{2} \\
d_{s}(t)=\frac{1}{2} \mathbf{E} \min \left\{t^{2} X_{s}^{2}, 1\right\}, \quad d_{0}(t)=\frac{1}{2} \mathbf{E} \min \left\{t^{2}|X-\mu(x)|^{2}, 1\right\} .
\end{gathered}
$$

Лемма 8. $v(t) \preceq d(t)$, и если имеет место неравенство (1), то $v(t) \simeq d(t)$. 
Д ок а з а тель с т в о. Имеем

$$
\begin{aligned}
v_{s}(t) & =\int_{0}^{\infty}(1-\cos x t) \mathbf{P}\left\{\left|X_{s}\right| \in d x\right\} \\
& \leqslant C_{1}\left(\int_{0}^{1 / t} t^{2} x^{2} \mathbf{P}\left\{\left|X_{s}\right| \in d x\right\}+\mathbf{P}\left\{\left|X_{s}\right| \geqslant \frac{1}{t}\right\}\right), \\
v_{s}(t) & \geqslant C_{2} \int_{0}^{1 / t} t^{2} x^{2} \mathbf{P}\left\{\left|X_{s}\right| \in d x\right\} .
\end{aligned}
$$

Откуда вытекает, что $v(t) \simeq v_{s}(t) \preceq d_{s}(t)$.

Если неравенство $(1)$ выполняется, то $v_{s}(t) \simeq d_{s}(t)$. В силу слабых неравенств симметризации (см. [12, гл. V, п. 17.1]) $d_{s}(t) \simeq d_{0}(t)$, и так как из леммы 7 вытекает, что $d_{0}(t) \simeq d(t)$, то получаем утверждения леммы.

Лемма 9. $d(t) \simeq J(t)=\int_{0}^{t} v(u) d u / t, u$ если $v(u) \simeq b(u)$, где $b(u)$ монотонная функиия, то $v(t) \simeq d(t) \simeq J(t)$.

Д о к а з а т е л с с в о. Используем обозначения доказательства леммы 8 , добавив обозначение $J_{s}(t)=\int_{0}^{t} v_{s}(u) d u / t$. Тогда

$$
\begin{aligned}
J_{s}(t) & =\frac{1}{t} \int_{0}^{t} \int_{0}^{\infty}(1-\cos u x) \mathbf{P}\left\{\left|X_{s}\right| \in d x\right\} d u \\
& =\int_{0}^{\infty}\left(1-\frac{\sin x t}{x t}\right) \mathbf{P}\left\{\left|X_{s}\right| \in d x\right\} \\
& \geqslant C_{1}\left(t^{2} \int_{0}^{1 / t} x^{2} \mathbf{P}\left\{\left|X_{s}\right| \in d x\right\}+\mathbf{P}\left\{\left|X_{s}\right| \geqslant \frac{1}{t}\right\}\right) .
\end{aligned}
$$

Следовательно, $d_{s}(t) \preceq J_{s}(t)$. В силу леммы $8 v_{s}(t) \preceq d_{s}(t), d_{s}(t)-$ монотонно неубывающая функция, и потому $J_{s}(t) \preceq d_{s}(t)$. Так как $v_{s}(t) \simeq v(t)$, то $J_{s}(t) \simeq J(t)$, кроме того, $d_{s}(t) \simeq d(t)$ (см. конец доказательства леммы 8), и потому $d(t) \simeq J(t)$. Если $v(t)$ почти монотонна, то $J(t) \preceq v(t)$, и так как $v(t) \preceq d(t)$, то $v(t) \simeq d(t) \simeq J(t)$.

Доказательство те о ремы 1. 1) $\Leftrightarrow 2$ ). Эквивалентность утверждений п. 1) и п. 2) теоремы является переформулировкой леммы работы [3].

1) $\Rightarrow 3)$. Если неравенство (1) выполняется, то $g(t) \simeq v(t) \simeq d(t)$ в силу леммы 8. Откуда с помощью леммы 3 получаем при некоторых постоянных $C_{1}>0$ и $C_{2}>0$, что $D\left(C_{1} t\right) \preceq G(t) \preceq D\left(C_{2} t\right)$, и так как $D$ - н. в 0 в. ф., то $D(t) \simeq G(t)$ и $G(t)-$ н. в 0 в. ф. Следовательно, в силу теоремы 5 и леммы $5 G(1 / x) \simeq \widehat{G}(x) \simeq q(x)$. Откуда и вытекает, что $Q(n) \simeq D(1 / n)$.

$3) \Rightarrow 2)$. Если $Q(n) \simeq D(1 / n)$, то в силу лемм 4 и 6

$$
\widehat{G}(2 x) \preceq q(x) \simeq D\left(x^{-1}\right) \preceq \widehat{G}(x) .
$$


Из леммы 8 следует, что $v(t) \simeq g(t) \preceq d(t)$, а тогда с помощью леммы 6 получаем, что $D(u) \preceq G\left(C_{1} u\right)$ при некоторой постоянной $C_{1}>0$. В этом случае из (3) следует, что $\widehat{G}(2 x) \preceq G\left(C_{1} / x\right)$, но из леммы 1 вытекает, что $G(C / x) \preceq \widehat{G}(2 x)$ при любой постоянной $C>0$, откуда получаем, что $G\left(2 C_{1} t\right) \preceq \widehat{G}\left(C_{1} t\right)$. Следовательно, $G(t)-$ н. в 0 в. ф., и в силу теоремы 5 $\widehat{G}(x)-$ н. на б. у. ф. Отсюда и из (3) следует, что $\widehat{G}(x) \simeq D(1 / x)$, и, значит, $D-$ н. в 0 в. ф.

Доказательст во теоремы 3. 1) $\Rightarrow 2$ ) и 3 ). Если $V-$ н. в 0 в. ф., то, так как $v(t) \simeq g(t)$, с помощью леммы 3 получим, что $V\left(C_{1} u\right) \preceq G(u) \preceq V\left(C_{2} u\right) \simeq V\left(C_{1} u\right)$, и, следовательно, $G(u) \simeq V(u)$. В силу теоремы 5 , так как $G(u)-$ н. в 0 в. $ф$., то $\widehat{G}(x)-$ н. на б. у. $ф$. и $\widehat{G}(x) \simeq G(1 / x)$. Из леммы 6 и соотношения $\widehat{G}(2 x) \simeq \widehat{G}(x)$ вытекает, что $q(x) \simeq \widehat{G}(x) \simeq G(1 / x)$, и, следовательно, $q(x)-$ н. на б. у. ф. и $Q(n) \simeq G(1 / n)$.

$2) \Rightarrow 1)$. Если $Q(m n) \simeq Q(n)$ при некотором $m=2,3, \ldots$, то из леммы 5 вытекает, что $q(x)-$ н. на. б. у. ф., и так как в силу леммы 6 $q(2 x) \preceq \widehat{G}(2 x) \preceq q(x) \simeq q(2 x)$, то $\widehat{G}(x)-$ н. на б. у. ф. Отсюда с помощью теоремы 5 получаем, что $G(u)-$ н. в 0 в. ф., и так как $g(t) \simeq v(t)$ (и потому $G$ и $V$ связаны оценками леммы 3 ), то и $V-$ н. в 0 в. ф.

$3) \Rightarrow 1)$. Если $Q(n) \simeq V(1 / n)$, то $q(x) \simeq V(1 / x)$ в силу леммы 4 , поэтому из леммы 6 вытекает, что $\widehat{G}(2 x) \preceq V(1 / x)$. Так как $v(t) \simeq g(t)$, то, применяя лемму 3 , получим $V(1 / x) \preceq G\left(C_{2} / x\right)$ при некоторой постоянной $C_{2}>0$. Таким образом, $\widehat{G}(2 x) \preceq G\left(C_{2} / x\right) \preceq \widehat{G}(C x)$, последнее соотношение имеет место при любой постоянной $C>0$ в силу леммы 1 , и, следовательно, $\widehat{G}(x)-$ н. на б. у. Ф. Из теоремы 5 вытекает, что $G(u)-$ н. в 0 в. ф., и так как $G$ и $V$ связаны оценками леммы 3 , то и $V(u)-$ н. в 0 в. $ф$.

Доказ а тель с т в о т е о ремы 2. Если $d(t)-$ п. в 0 в. ф., то $D(u)-$ н. в 0 в. ф., и обратно, если $D(u)-$ н. в 0 в. ф., то $d(t)-$ п. в 0 в. ф. Эти утверждения является следствиями леммы 1 работы [7]. Таким образом, п. 1) теоремы имеет место одновременно с любым утверждением теоремы 1.

$2) \Rightarrow 1)$. Если $v(t) \simeq a(t)-$ п. в 0 в. ф., то с помощью леммы 9 получаем, что $v(t) \simeq d(t)$, и, следовательно, в силу леммы $2 d(t)-$ п. в 0 в. ф.

$1) \Rightarrow 2)$. При выполнении неравенства $(1) v(t) \simeq d(t)$ в силу леммы 8 , и $d(t)-$ п. в. 0 в. ф., поэтому $v(t) \simeq d(t)-$ п. в 0 в. $ф$.

$3) \Rightarrow 1)$. Пусть $v(t) \simeq b(t)$, где $b(t)-$ монотонная функция, и $Q(m n) \simeq Q(n)$ при некотором $m \in\{2,3, \ldots\}$. С помощью леммы 9 получаем, что $v(t) \simeq d(t)$. Поэтому из леммы 3 вытекает, что $V\left(C_{1} u\right) \preceq$ $D(u) \preceq V\left(C_{2} u\right)$, откуда, поскольку в силу теоремы $3 V(u)-$ н. в 0 в. ф., получаем, что $D(u)-$ н. в 0 в. ф. 
1) $\Rightarrow 3)$. При выполнении неравенства (1) в силу теоремы 1 $Q(n) \simeq D(1 / n)$ и $D(t)$ - н. в 0 в. ф., поэтому $Q(n m) \simeq Q(n)$ при любом $m=2,3, \ldots$.

3. Доказательство теоремы 4. Приведем необходимые для доказательства утверждения относительно порядка изменения н. на б. у. ф.

Лемма 10. Если $a_{1}(x)$ - н. на б. у. $\oint ., a_{1}(x) \simeq a_{2}(x)$ u $a_{2}-$ монотонная функиия, то $a_{2}$ - н. на б. у. Ф. и порядки надстепенного изменения функиий $a_{1}$ и $a_{2}$ совпадают.

Д ок аз а т ль с т о. Утверждение, что $a_{2}$ - н. на б. у. ф., вытекает непосредственно из определения. При $i=1,2$ и $y \geqslant 1$ положим $B_{i}(y)=\lim \sup _{x \rightarrow \infty} a_{i}(x) / a_{i}(x y), H_{i}(z)=\ln B_{i}\left(e^{z}\right)-$ конечная полуаддитивная при $z \geqslant 0$ функция. Из условия $a_{1}(x) \simeq a_{2}(x)$ следует, что $B_{1}(y) \simeq B_{2}(y)$, и потому при $i=1,2$ для $\alpha_{i}=\lim _{z \rightarrow \infty} H_{i}(z) / z=$ $\lim _{y \rightarrow \infty} \ln B_{i}(y) / \ln y$ получаем, что $\alpha_{1}=\alpha_{2}$, т.е. порядки надстепенного изменения $\alpha_{1}$ и $\alpha_{2}$ совпадают.

Лемма 11. Если $q(x)$ - н. на б. у. ф. с порядком надстепенного изменения $-\alpha$, то $\alpha>\frac{1}{2}$.

Д о к а з а т е л с т в о. Заметим, что при $m=1,2, \ldots$

$$
B(m)=\limsup _{x \rightarrow \infty} \frac{q(x)}{q(x m)}=\limsup _{n \rightarrow \infty} \frac{Q(n)}{Q(n m)} .
$$

(Действительно, так как при $x \geqslant 1$ справедливы неравенства $Q([x]+1) /$ $Q([x] m) \leqslant q(x) / q(x m) \leqslant Q([x]) / Q(([x]+1) m)$, то (4) получается из предельной теоремы для отношений функций концентрации [9].) Таким образом,

$$
\alpha=\lim _{z \rightarrow \infty} \frac{H(z)}{z}=\lim _{m \rightarrow \infty} \frac{\ln B(m)}{\ln m}
$$

где $H(z)=\ln B\left(e^{z}\right)$ - конечная полуаддитивная функция (см. доказательство леммы П4 из [5]).

Для доказательства оценки $\alpha \geqslant \frac{1}{2}$ воспользуемся следующим неравенством (см. [8]):

$$
Q\left(h, F^{(n m)}\right) \leqslant \frac{A Q\left(h, F^{(n)}\right)}{\left(m\left(1-Q\left(h, F^{(n)}\right)\right)\right)^{1 / 2}},
$$

где $A$ - абсолютная постоянная. Поскольку $\lim _{n \rightarrow \infty} Q\left(h, F^{(n)}\right)=0$, из (6) вытекает неравенство $B(m) \geqslant m^{1 / 2} / A$. Откуда с помощью (5) получаем неравенство $\alpha \geqslant \frac{1}{2}$.

Лемма 12. 1) Если соотношение

$$
\lim _{m \rightarrow \infty} m^{-\beta} \limsup _{n \rightarrow \infty} \frac{Q(n)}{Q(n m)}=0
$$


выполняется, то для - $\alpha-$ порядка надстепенного изменения функиии $g$ - имеет место неравенство $\alpha<\beta$.

2) Если порядок изменения функиии q равен $-\alpha$, то соотношение (7) выполняется при любом $\beta>\alpha$.

Д о к а з а т е л ь с т в о. Если имеет место (7), то $\lim _{m \rightarrow \infty} m^{-\beta} \times$ $B(m)=0$, и, следовательно, $B\left(m_{0}\right)=C_{1} m_{0}^{\beta}$ при некотором $m_{0}$, где $0<$ $C_{1}<1$, и $H\left(\ln m_{0}\right)=\beta \ln m_{0}+\ln C_{1}$. В соответствии с теоремой 7.6.1 из [13] $\alpha=\lim _{z \rightarrow \infty} H(z) / z=\inf _{z>0} H(z) / z \leqslant H\left(\ln m_{0}\right) / \ln m_{0}<\beta$.

Для доказательства п. 2) леммы заметим, что при $\alpha<\beta_{1}<\beta$ и при всех $z \geqslant z_{0}$ выполнено неравенство $H(z) \leqslant \beta_{1} z$, и потому $B(y) \leqslant y^{\beta_{1}}$ при $y \geqslant y_{0}$; отсюда и из (5) и следует утверждение п. 2) леммы.

Перейдем непосредственно к доказательству теоремы 4. Сначала установим, что если распределение симметрично относительно 0 и порядок надстепенного изменения последовательности $\{Q(n), n=1,2 \ldots\}$ равен $-\alpha$, то порядок некомпактности $\left\{F^{(n)}\right\}$ не превосходит $k=[2 \alpha-1]$. Для этого воспользуемся схемой доказательства теоремы 3 из работы[8].

Пусть доказываемое утверждение не имеет места, тогда существуют последовательность целых чисел $\{n(j), j=1,2, \ldots\}$ и набор $0<p_{1}<\cdots<p_{k+1}<1$ такие, что при $i=1, \ldots, k+1$ последовательность распределений случайных величин $S(n(j)) / K\left(p_{i}, n(j)\right)$ при $j \rightarrow \infty$ слабо сходится к предельному несобственному распределению $G_{i}$ и $0<G_{1}(\mathbf{R})<\cdots<G_{k+1}(\mathbf{R})<1$. При этом можно выбрать $p_{1}, \ldots, p_{k+1}$ такими, что

$$
0<p_{1} \leqslant G_{1}(\mathbf{R})<p_{2} \leqslant G_{2}(\mathbf{R})<\cdots<p_{k+1} \leqslant G_{k+1}(\mathbf{R}) .
$$

Действительно, если $p_{i+1}=G_{i}(\mathbf{R})$ при некотором $i=1, \ldots, k+1$, то $p_{i+1}<G_{i+1}(\mathbf{R})$, и потому, если выбрать $p_{i+1}^{\prime}$ так, что

$$
p_{i+1}<p_{i+1}^{\prime}<G_{i+1}(\mathbf{R}),
$$

то $K\left(p_{i+1}^{\prime}, n(j)\right) \simeq K\left(p_{i+1}, n(j)\right), j \rightarrow \infty$. Следовательно, из совокупности $p_{1}, \ldots, p_{i-1}, p_{i}^{\prime}, p_{i+1}, \ldots, p_{k+1},-$ при необходимости переходя к подпоследовательности $\{n(j), j=1,2, \ldots\}$, чтобы обеспечить сходимость к предельному распределению, - получим последовательность и набор с указанными свойствами. При этом нетрудно обнаружить, что выполняются соотношения

$$
\lim _{j \rightarrow \infty} \frac{K\left(p_{i}, n(j)\right)}{K\left(p_{i+1}, n(j)\right)}=0, \quad i=1, \ldots, k .
$$

При $i=1, \ldots, k+1$ и $j=1,2, \ldots$ обозначим

$$
K_{i}(j)=K\left(p_{i}, n(j)\right),
$$


и пусть $F_{i j}$ - распределения такие, что для любого борелевского множества $A$

$$
\begin{aligned}
F_{1, j}(A)= & \mathbf{P}\left\{S(n(j)) \in A \mid S(n(j))<K_{1}(j)\right\}, \\
F_{i, j}(A)= & \mathbf{P}\left\{S(n(j)) \in A \mid K_{i-1}(j)\right. \\
\left.\leqslant S(n(j))<K_{i}(j)\right\}, \quad i=2, \ldots, k+1, & \\
F_{k+2, j}(A)= & \mathbf{P}\left\{S(n(j)) \in A \mid S(n(j)) \geqslant K_{k+1}(j)\right\},
\end{aligned}
$$

и

$$
\begin{aligned}
r_{1}(j) & =\mathbf{P}\left\{S(n(j))<K_{1}(j)\right\}, \\
r_{i}(j) & =\mathbf{P}\left\{K_{i-1}(j) \leqslant S(n(j))<K_{i}(j)\right\}, \quad i=2, \ldots, k+1, \\
r_{k+2}(j) & =\mathbf{P}\left\{S(n(j)) \geqslant K_{k+1}(j)\right\} .
\end{aligned}
$$

Тогда имеют место соотношения

$$
\begin{gathered}
F^{(1)}=\sum_{i=1}^{k+2} r_{i}(j) F_{i, j}, \\
\lim _{j \rightarrow \infty} r_{i}(j)=p_{i}-p_{i-1}=q_{i}>0, \quad Q\left(h, F_{i, j}\right) \leqslant \frac{Q\left(h, F^{(n(j))}\right)}{r_{i}(j)},
\end{gathered}
$$

где $i=1, \ldots, k+2$ и $p_{0}=0, p_{k+2}=1$.

Докажем, что при любом фиксированном $m$ и $i=2, \ldots, k+2$

$$
\limsup _{j \rightarrow \infty} Q\left(m K_{i-1}(j), F_{i, j}\right) \leqslant \lambda<1 .
$$

Сушествует интервал $\left[y, y+2 m K_{i-1}(j)\right]$, для которого $F_{i, j}([y, y+$ $\left.\left.2 m K_{i-1}(j)\right]\right) \geqslant Q\left(m K_{i-1}(j), F_{i, j}\right)$. Если 0 принадлежит интервалу $\left[y, y+2 m K_{i-1}(j)\right]$, то $F_{i, j}\left[y, y+2 m K_{i-1}(j)\right] \leqslant\left(G_{i-1}(\mathbf{R})-p_{i-1}\right) / q_{i}+\varepsilon(j)$, $\lim _{j \rightarrow \infty} \varepsilon(j)=0$. Если 0 не принадлежит этому интервалу, то $F_{i, j}[y, y+$ $\left.2 m K_{i-1}(j)\right] \leqslant \frac{1}{2}$. Следовательно,

$$
\lambda \leqslant \max \left\{\frac{1}{2}, \frac{G_{1}(\mathbf{R})-p_{1}}{q_{2}}, \ldots, \frac{G_{k+1}(\mathbf{R})-p_{k+1}}{q_{k+2}}\right\}<1 .
$$

Пусть $m>1$. Так как имеет место (9), то

$F^{(m n(j))}=\sum_{l_{1}=0}^{m} \cdots \sum_{l_{k+2}=1}^{m} F_{1, j}^{\left(l_{1}\right)} * \cdots * F_{k+2, j}^{\left(l_{k+2}\right)} \mathbf{P}\left\{\xi_{1}=l_{1}, \ldots, \xi_{k+2}=l_{k+2}\right\}$

где $G * H$ - свертка вероятностных распределений $G$ и $H$ и $\left(\xi_{1}, \ldots, \xi_{k+2}\right)$ - случайный вектор с полиномиальным распределением: при $0 \leqslant l_{i} \leqslant m, i=1, \ldots, k+2, l_{1}+\cdots+l_{k+2}=m$

$$
\mathbf{P}\left\{\xi_{1}=l_{1}, \ldots, \xi_{k+2}=l_{k+2}\right\}=\frac{m !}{l_{1} ! \cdots l_{k+2} !} r_{1}(j)^{l_{1}} \cdots r_{k+2}(j)^{l_{k+2}} .
$$


Лемма 13. Если $G$ и $H$ - вероятностнье распределения и при $a>0$ выполнено $G([-a, a])=1, \operatorname{mo} Q(h, G * H) \leqslant Q(h, G) Q(2 a+h, H)$.

Д ок аз а т е л с т в о. Пусть $A=[-h / 2, h / 2]$. Тогда

$$
\begin{array}{rl}
G & * H(A+z)=\int_{\mathbf{R}} G(A+z-y) H(d y) \\
& =\int_{[z-h / 2-a, z+h / 2+a]} G(A+z-y) H(d y) \leqslant Q(h, G) Q(2 a+h, H),
\end{array}
$$

так как $G(A+z-y)=0$, если пересечение интервалов $A+z-y$ и $[-a, a]$ пусто.

Применим эту лемму для оценки функций концентрации распределений $F_{1, j}^{\left(l_{1}\right)} * \cdots * F_{k+2, j}^{\left(l_{k+2}\right)}$. Положим

$$
M_{i}=l_{1} K_{1}(j)+\cdots+l_{i} K_{i}(j), \quad i=1, \ldots, k+1,
$$

тогда

$$
\begin{aligned}
Q\left(h, F_{1, j}^{\left(l_{1}\right)} * \cdots * F_{k+2, j}^{\left(l_{k+2}\right)}\right) & \leqslant Q\left(h, F_{1, j}^{\left(l_{1}\right)} * \cdots * F_{k+1, j}^{\left(l_{k+1}\right)}\right) Q\left(2 M_{k+1}+h, F_{k+2, j}^{\left(l_{k+2}\right)}\right) \\
& \leqslant Q\left(h, F_{1, j}^{\left(l_{1}\right)}\right) \cdots Q\left(2 M_{k+1}+h, F_{k+2, j}^{\left(l_{k+2}\right)}\right) .
\end{aligned}
$$

Для оценки $Q\left(2 M_{i-1}+h, F_{i, j}^{\left(l_{i}\right)}\right)$ при $i=2, \ldots, k+2$ и $l_{i} \geqslant 1$ воспользуемся следуюшим неравенством (см. [14]): при некоторой постоянной $A_{1}>0$

$$
Q\left(2 M_{i-1}+h, F_{i, j}^{\left(l_{i}\right)}\right) \leqslant \frac{A_{1}}{\left(l_{i}\left(1-Q\left(2 M_{i-1}+h, F_{i, j}\right)\right)\right)^{1 / 2}} \leqslant \frac{2 A_{1}}{\left(l_{i}(1-\lambda)\right)^{1 / 2}}
$$

для всех достаточно больших $j$. При этом $Q\left(2 M_{i-1}+h, F_{i, j}\right)$ оценивается с помощью (11), так как в силу соотношений (8) $2 M_{i-1}+h \leqslant 3 l_{i-1} K_{i-1}(j)$ при всех достаточно больших $j$.

Для оценки $Q\left(h, F_{1, j}^{\left(l_{1}\right)}\right)$ воспользуемся неравенством $(6)$, доказанным в [8]: при $l_{1} \geqslant 1$

$$
Q\left(h, F_{1, j}^{\left(l_{1}\right)}\right) \leqslant \frac{A Q\left(h, F_{1, j}\right)}{\left(l_{1}\left(1-Q\left(h, F_{1, j}\right)\right)\right)^{1 / 2}} \leqslant \frac{2 A Q\left(h, F^{(n(j)}\right)}{q_{1} l_{1}^{1 / 2}}
$$

для всех достаточно больших $j$, так как $\lim _{j \rightarrow \infty} q\left(h, F_{1, j}\right)=0$ и имеют место соотношение (10).

Таким образом, если $l_{i} \geqslant 1, i=1, \ldots, k+2$, то, подставляя (14) и (15) в неравенство (13), получим

$$
Q\left(h, F_{1, j}^{\left(l_{1}\right)} * \cdots * F_{k+2, j}^{\left(l_{k+2}\right)}\right) \leqslant C_{1} Q\left(h, F^{(n(j))}\right)\left(l_{1} \cdots l_{k+2}\right)^{-1 / 2}
$$


при всех достаточно больших $j$. Подставляя эти оценки в оценку для $Q\left(h, F^{(m n(j))}\right)$, вытекающую из равенства (12), получим при $m q_{i}>4$, $i=1, \ldots, k+2$, для всех достаточно больших $j$

$$
\begin{aligned}
Q\left(h, F^{(m n(j))}\right) \leqslant & C_{1} Q\left(h, F^{(n(j))}\right) m^{-(k+2) / 2} \\
& \times \mathbf{E}\left(\left(\frac{m}{\xi_{1}} \cdots \frac{m}{\xi_{k+2}}\right)^{1 / 2} ; \xi_{i} \geqslant \frac{m r_{i}(j)}{2}, i=1, \ldots, k+2\right) \\
& +C_{2} Q\left(h, F^{(n(j))}\right) \sum_{i=1}^{k+2} \mathbf{P}\left\{\xi_{i}<2^{-1} m r_{i}(j)\right\}
\end{aligned}
$$

где положительные постоянные $C_{1}, C_{2}$ не зависят от $m$ и $j$. При этом если хотя бы одно значение $\xi_{i}$ меньше $m r_{i}(j) / 2$, то используется очевидная оценка

$$
Q\left(h, F_{1, j}^{\left(l_{1}\right)} * \cdots * F_{k+2, j}^{\left(l_{k+2}\right)}\right) \leqslant Q\left(h, F_{i_{0}, j}\right) \leqslant 2 Q\left(h, F^{(n(j))}\right) / q_{i_{0}} .
$$

Первое неравенство имеет место при любом значении $i_{0}$, для которого $l_{i_{0}} \geqslant 1$, а второе в силу соотношений $(10)$ - при всех достаточно больших $j$.

Случайная величина $\xi_{i}, i=1, \ldots, k+2$, имеет биномиальное распределение с числом испытаний $m$ и вероятностью успеха $r_{i}(j)$, и потому с помощью неравенства Чебышева с показательной функцией (см. [15, c. 207]) получаем оценку $\mathbf{P}\left\{\xi_{i}<m r_{i}(j) / 2\right\} \leqslant \exp \left\{-C_{3} m\right\}$. Таким образом, из неравенства (16) вытекает, что при всех достаточно больших $j$

$$
Q\left(h, F^{(m n(j))}\right) \leqslant C_{4} Q\left(h, F^{(n(j))}\right) m^{-(k+2) / 2}+C_{5} Q\left(h, F^{(n(j))}\right) \exp \left\{-C_{3} m\right\}
$$

где положительные постоянные $C_{3}, C_{4}, C_{5}$ не зависят от $m$ и $j$. Следовательно,

$$
\liminf _{m \rightarrow \infty} m^{-(k+2) / 2} \liminf _{j \rightarrow \infty} \frac{Q\left(h, F^{(n(j))}\right)}{Q\left(h, F^{(m n(j))}\right)} \geqslant \frac{1}{C_{4}}>0,
$$

но так как $(k+2) / 2>\alpha$, то в силу п. 2) леммы 12

$$
\lim _{m \rightarrow \infty} m^{-(k+2) / 2} \limsup _{n \rightarrow \infty} \frac{Q\left(h, F^{(n)}\right)}{Q\left(h, F^{(m n)}\right)}=0 .
$$

Таким образом, мы пришли к противоречию, и, следовательно, утверждение теоремы доказано, если распределение $X$ симметрично относительно 0.

В качестве следствия доказанного утверждения получим, что семейство $\left\{F_{s}^{(n)}, n=1,2, \ldots\right\}$, где $F_{s}^{(1)}$ - распределение $X_{s}$, в условиях теоремы имеет конечный порядок некомпактности, не превосходящий $k=[2 \alpha-1]$. Действительно, для $Q_{s}(n)=Q\left(h, F_{s}^{(n)}\right), n=1,2, \ldots$, имеет 
место соотношение $Q(4 n) \preceq Q_{s}(n) \preceq Q(n)$ [11, гл. III, §1]. Следовательно, так как $q-$ н. на б. у. $ф$., то $q_{s}(x) \simeq q(x)$ и порядки изменения $q_{s}(x)$ и $q(x)$ совпадают в силу леммы 10 . Откуда следует, что последовательность $\left\{F_{s}^{(n)}, n=1,2, \ldots\right\}$ имеет порядок некомпактности, не больший $k=[2 \alpha-1]$.

Теперь перейдем к доказательству теоремы в общем случае. Допустим, что утверждение теоремы не имеет места, тогда существуют последовательность $\{n(j), j=1,2, \ldots\}$ и набор $0<p_{1}<\cdots<$ $p_{k+1}<1$ такие, что последовательность распределений случайных величин $(S(n(j))-\mu(n(j))) / K\left(p_{i}, n(j)\right)$ слабо сходится при $j \rightarrow \infty$ к несобственному распределению $G_{i}, i=1, \ldots, k+1$, и $0<G_{1}(\mathbf{R})<\cdots<$ $G_{k+1}(\mathbf{R})<1$. При этом можно считать, что

$$
0<p_{1} \leqslant G_{1}(\mathbf{R})<p_{2} \leqslant G_{2}(\mathbf{R})<\cdots<p_{k+1} \leqslant G_{k+1}(\mathbf{R})
$$

(см. начало доказательства теоремы 4). Кроме того, при $i=2, \ldots, k+1$ для $p_{i}^{\prime}\left(G_{i-1}(\mathbf{R})<p_{i}^{\prime} \leqslant G_{i}(\mathbf{R})\right)$

$$
\lim _{j \rightarrow \infty} \frac{K\left(p_{i-1}, n(j)\right)}{K\left(p_{i}^{\prime}, n(j)\right)}=0 .
$$

Рассмотрим при $i=1, \ldots, k+1$ последовательность распределений случайных величин $S_{s}(n(j)) / K\left(p_{i}, n(j)\right)$, где $S_{s}(n)=S(n)-S^{\prime}(n)$, $S^{\prime}(n)=\sum_{i=1}^{n} X^{\prime}(i)$ и последовательности $\left\{X^{\prime}, X^{\prime}(1), X^{\prime}(2), \ldots\right\}$ и $\{X, X(1), X(2), \ldots\}$ одинаково распределены и независимы. При необходимости переходя к подпоследовательности последовательности $\{n(j), j=1,2, \ldots\}$, получим, что при $i=1, \ldots, k+1$ последовательность распределений $S_{s}(n(j)) / K\left(p_{i}, n(j)\right)$ слабо сходится к (возможно, несобственному) распределению $G_{i}^{\prime}$ и $0 \leqslant G_{1}^{\prime}(\mathbf{R}) \leqslant \cdots \leqslant G_{k+1}^{\prime}(\mathbf{R}) \leqslant 1$.

Докажем, что

$$
0<G_{1}^{\prime}(\mathbf{R})<\cdots<G_{k+1}^{\prime}(\mathbf{R})<1 .
$$

Нетрудно заметить, что

$$
G_{1}^{\prime}(\mathbf{R}) \geqslant\left(G_{1}(\mathbf{R})\right)^{2}>0 .
$$

Из слабых неравенств симметризации (см. [12, гл. V, п. 17.1]) вытекает

$$
\mathbf{P}\left\{\left|S_{s}(n(j))\right|<x K\left(p_{i}, n(j)\right)\right\} \leqslant \frac{1+\mathbf{P}\left\{|S(n(j))-\mu(n(j))|<x K\left(p_{i}, n(j)\right)\right\}}{2}
$$

и потому

$$
G_{i}^{\prime}(\mathbf{R}) \leqslant \frac{G_{i}^{\prime}(\mathbf{R})+1}{2}<1 \quad \text { при } \quad i=1, \ldots, k+1 .
$$


Далее, для $i=1, \ldots, k$ и $y>0$ такого, что $G_{i}^{\prime}((-y, y))>0$, имеем

$$
\begin{aligned}
& G_{i+1}^{\prime}(\mathbf{R})-G_{i}^{\prime}(\mathbf{R}) \\
& \geqslant \underset{j \rightarrow \infty}{\limsup } \mathbf{P}\left\{2 K\left(p_{i+1}, n(j)\right) \geqslant\left|S_{s}(n(j))\right|>\frac{K\left(p_{i+1}^{\prime}, n(j)\right)}{2}\right\} \\
& \geqslant \underset{j \rightarrow \infty}{\limsup } \mathbf{P}\left\{K\left(p_{i+1}, n(j)\right) \geqslant|S(n(j))-\mu(n(j))|>K\left(p_{i+1}^{\prime}, n(j)\right),\right. \\
& \left.\left|S^{\prime}(n(j))-\mu(n(j))\right| \leqslant y K\left(p_{i}, n(j)\right)\right\} \\
& \geqslant\left(p_{i+1}-p_{i+1}^{\prime}\right) G_{i}^{\prime}((-y, y))>0,
\end{aligned}
$$

где $p_{i+1}^{\prime}$ выбирается удовлетворяющим неравенствам $G_{i}(\mathbf{R})<p_{i+1}^{\prime}<$ $p_{i+1}$, что дает возможность в приведенных выше оценках использовать соотношения (17). Таким образом,

$$
0<G_{1}^{\prime}(\mathbf{R})<\cdots<G_{k+1}^{\prime}(\mathbf{R})<1 .
$$

Выберем $p_{s, 1}, \ldots, p_{s, k+1}$ такими, что $0<p_{s, 1}<G_{1}^{\prime}(\mathbf{R})$ и $G_{i-1}^{\prime}(\mathbf{R})<$ $p_{s, i}<G_{i}^{\prime}(\mathbf{R})$ при $i=2, \ldots, k+1$. При необходимости переходя к подпоследовательности последовательности $\{n(j), j=1,2, \ldots\}$ для обеспечения слабой сходимости соответствующих распределений, получим, что при $i=1, \ldots, k+1$ последовательность распределений случайных величин $S_{s}(n(j)) / K_{s}\left(p_{s, i}, n(j)\right)$ слабо сходится при $j \rightarrow \infty$ к несобственному распределению $G_{s, i}$ и

$0<G_{s, 1}(\mathbf{R}) \leqslant G_{1}^{\prime}(\mathbf{R})<G_{s, 2}(\mathbf{R}) \leqslant G_{2}^{\prime}(\mathbf{R})<\cdots<G_{s, k+1}(\mathbf{R}) \leqslant G_{k+1}^{\prime}(\mathbf{R})<1$.

Так как $0<p_{s, 1} \leqslant G_{s, 1}(\mathbf{R}) \leqslant G_{1}^{\prime}(\mathbf{R})$ и $G_{i-1}^{\prime}(\mathbf{R})<p_{s, i} \leqslant G_{s, i}(\mathbf{R}) \leqslant G_{i}^{\prime}(\mathbf{R})$ при $i=2, \ldots, k+1$, то для последовательности $\{n(j), j=1,2, \ldots\}$ и набора $p_{s, 1}, \ldots, p_{s, k+1}$ последовательность распределений случайных величин $S_{s}(n(j)) / K_{s}\left(p_{s, i}, n(j)\right)$ слабо сходится при $j \rightarrow \infty$ к несобственному распределению $G_{s, i}, i=1, \ldots, k+1$, и

$$
0<G_{s, 1}(\mathbf{R})<\cdots<G_{s, k+1}(\mathbf{R})<1
$$

т.е. последовательность $\left\{F_{s}^{(n)}, n=1,2, \ldots\right\}$ не является конечно некомпактной порядка, не превосходящего числа $k$, что противоречит ранее доказанному утверждению. Полученное противоречие завершает доказательство теоремы 4.

Д ок азатель с тв о сл е д ствия. Необходимо, в соответствии с п. 3) теоремы 2, установить, что для одновершинного распределения $F^{(1)}$ справедливы соотношения $v(t) \simeq v_{s}(t) \simeq b(t), b(t)-$ монотонная неубывающая функция. Для этого докажем лемму.

Лемма 14. Если $F^{(1)}$ - одновершинное распределение, то $F_{s}^{(1)}$ также одновершинное распределение. 
Д о к а з а т ел ь с т в о. Пусть сначала распределение $F^{(1)}$ имеет плотность

$$
p(x)=\sum_{k=1}^{N} a_{k} I_{A_{k}}(x),
$$

где $A_{1}, \ldots, A_{N}$ - отрезки, для которых $0 \in A_{1} \subset \cdots \subset A_{N}$, и $I_{A}(x)-$ индикатор множества $A$. Покажем, что плотность $F_{s}^{(1)}$, являюшаяся сверткой плотностей $p * p_{1}(x)$, где $p_{1}(x)=\sum_{k=1}^{N} a_{k} I_{B_{k}}(x)$ и $B_{k}=-A_{k}$, $k=1, \ldots, N$, есть плотность одновершинного распределения.

Квадрат $A_{k} \times B_{k}=\left\{(x, y): x \in A_{k}, y \in B_{k}\right\}$ имеет диагональ $C_{k}$, которая лежит на прямой $x+y=0$. При $j \geqslant k$ диагональ $C_{k}$ содержится также в прямоугольнике $A_{k} \times B_{k}$, и потому свертка $I_{A_{k}} * I_{B_{j}}$ достигает максимума в 0 и является монотонно неубывающей (невозрастающей) функцией слева (справа) от 0 . При $k>j$ для квадрата $A_{j} \times B_{j}$ и прямоугольника $A_{k} \times B_{j}$ используется та же аргументация. Поэтому свертка $I_{A_{k}} * I_{B_{j}}$ достигает максимума в 0 и является монотонно неубывающей (невозрастающей) функцией слева (справа) от 0 . Таким образом, свертка $p * p_{1}(x)$ является монотонно неубывающей (невозрастающей) функцией слева (справа) от 0 , т.е. $F_{s}^{(1)}$ - одновершинное распределение.

Используя схему приближения [16] одновершинного распределения $F^{(1)}$ распределениями приведенного выше специального вида, получим, что $F_{s}^{(1)}$ - одновершинное распределение и в общем случае.

В соответствии с теоремой Хинчина (см. $[17$, гл. $6, \S 32]) v_{s}(t)=$ $\int_{0}^{t} h(u) d u / t$, где $1-h(u)$ - характеристическая функция распределения симметричной случайной величины $Y$, и потому при некоторой постоянной $C_{1}$

$$
\begin{aligned}
v_{s}(t) & =\frac{1}{t} \int_{0}^{t} \int_{0}^{\infty}(1-\cos x u) \mathbf{P}\{|Y| \in d x\} d u \\
& =\int_{0}^{\infty}\left(1-\frac{\sin x t}{x t}\right) \mathbf{P}\{|Y| \in d x\} \\
& \geqslant C_{1}\left(t^{2} \int_{0}^{1 / t} x^{2} \mathbf{P}\{|Y| \in d x\}+\mathbf{P}\left\{|Y| \geqslant \frac{1}{t}\right\}\right) \\
& =2 C_{1} t^{2} \int_{0}^{1 / t} x \mathbf{P}\{|Y|>x\} d x=2 C_{1} d_{y}(t) .
\end{aligned}
$$

Из доказательства теоремы Хинчина вытекает, что

$$
2 F_{s}^{(1)}([x, \infty)) \leqslant \mathbf{P}\{|Y| \geqslant x\},
$$

и потому $d_{s}(t) \leqslant d_{Y}(t)$. Отсюда, привлекая оценку $(18)$, получим $d_{s}(t) \preceq$ $v_{s}(t)$. Из леммы 8 в свою очередь вытекает, что $v_{s}(t) \preceq d_{s}(t)$, и потому $v_{s}(t) \simeq d_{s}(t), d_{s}(t)-$ монотонная функция. 


\section{СПИСОК ЛИТЕРАТУРЫ}

1. Doeblin $W$. Sur l'ensemble de puissances d'une loi de probabilité. - Studia Math., 1940, v. 9, p. 71-96.

2. Feller $W$. On regular variation and local limit theorems. - Proceedings of the Fifth Berkeley Symposium on Mathematical Statistics and Probability. V. II. Contributions to Probability Theory. Part 1. Berkeley: Univ. of California Press, 1967, p. 373-388.

3. Hall P. Order of magnitude of the concentration function. - Proc. Amer. Math. Soc., 1983 , v. 89, № 1, p. 141-144.

4. Хенгартнер B., Теодореску Р. Функции концентрации. М.: Наука, 1980, 176 с.

5. Сенета E. Правильно меняюшиеся функции. М.: Наука, 1985, 141 с.

6. Боровков $A$. A. Вероятностные процессы в теории массового обслуживания. М.: Наука, 1972, 368 c.

7. Рогозин Б. А. Тауберовская теорема для мажорируемо меняюшихся возрастающих функций. - Сиб. матем. журн., 2002, т. 43, № 2, с. 442-445.

8. Kesten $H$. A sharper form of the Doeblin-Lévy-Kolmogorov-Rogozin inequality for concentration functions. - Math. Scand., 1969, v. 25, № 1, p. 133-144.

9. Рогозин Б.А. Предельная теорема для отношений функций концентрации. Теория вероятн. и ее примен., 2001, т. 46 , в. 4, с. 801-803.

10. Рогозин Б. А. Тауберовы теоремы для мажорируемо меняющихся убывающих функций. - Теория вероятн. и ее примен., 2002, т. 47 , в. 2, с. 357-366.

11. Петров В. В. Суммы независимых случайных величин. М.: Наука, 1972, 416 с.

12. Лоэв М. Теория вероятностей. М.: ИЛ, 1962, 719 с.

13. Хилле Э., Филлипс Р. Функциональный анализ и полугруппы. М.: ИЛ, 1962, $829 \mathrm{c}$.

14. Рогозин Б. А. Об одной оценке функций концентраций. - Теория вероятн. и ее примен., 1961, т. 6, в. 1, с. 103-105.

15. Боровков A. A. Теория вероятностей. М.: Наука, 1986, 431 с.

16. Рогозин Б. А. О максимуме плотности вероятности суммы случайных величин с одновершинными распределениями. - Литов. матем. сб., 1965, т. 5, № 3, с. 499503.

17. Гнеденко Б.В., Колмогоров А.Н. Предельные распределения для сумм независимых случайных величин. М.-Л.: Гостехиздат, 1949, 264 с.

Поступила в редакцию 15.VI.2001 